\title{
The Topics of Psychotherapy Research: An Analysis Based on Keywords
}

\author{
Alessandro Gennaro $^{1 凶}$, Claudia Venuleo ${ }^{1}$, Andrea F. Auletta ${ }^{1}$, and Sergio Salvatore
}

\begin{abstract}
A content analysis of the representative Journals in the field of psychotherapy research has been performed. The analysis focused on the articles' keywords. We analyzed 7,086 works published in 17 Journals, in the period 2005-2011, using a two-step multidimensional procedure. Firstly, a cluster analysis led to the extrapolation of 4 groups of keywords, each of them interpreted as the marker of a topic active within the literature. Secondly, a factorial analysis was carried out in order to picture the thematic orientation of the most representative Journals, namely the main topics they focus on and how they differ from each other in this respect.
\end{abstract}

Keywords: psychotherapy research, content analysis, key words, topics

Psychotherapy research is a dynamic, broad, and variegated area of investigation. Hundreds of works are produced yearly, spreading over a large range of subjects, foci of analysis, theoretical models and methodologies. Such heterogeneity makes it hard to form a comprehensive vision of the state of the art in this field (Manzo, 2010).

This paper intends to contribute to such a task. It provides a map of the main topics in the psychotherapy research area, the relation between them and their distribution among Journals. In so doing, our aim is twofold. On the one hand, our purpose is to provide an empirical analysis of the semantic context characterizing the current state of the field. On the other hand, our intention is to provide an empirical picture of the thematic orientation of the most representative Journals in the field, namely the main topics they focus on and how they differ from each other in this respect. More in particular, the analysis is oriented by the following three main questions:

a) Is the psychotherapy research a single field or does it appear to be a kind of "confederation" of separate areas of investigation having little, if any, overlap with each other?

\footnotetext{
${ }^{1}$ Department of History, Society and Human Studies, University of Salento, Italy.

Correspondence concerning this article should be addressed to Alessandro Gennaro, Palazzo Parlangeli, via Stampacchia 45, 73100 Lecce (LE).

Italy. Email: alessandro.gennaro@unisalento.it
}

b) However broad the field's inner differentiation is, how can it be interpreted: as a matter of thematic pluralism or as the result of deeper differentiation, concerning paradigmatic orientation, research goals and the like?

c) How is the research field's articulation represented by the Journals' orientation? Namely, do Journals tend to encompass the differentiations or do they tend to commit to specific sub-areas of the field?

It is worth highlighting the conceptual, methodological and practical interest of these questions. At the conceptual level, one has to consider that any topic is not a neutral fact; rather, it acquires meaning in terms of a particular theoretical framework (Salvatore, 2011). Consequently, the detection of themes of clinical research can tell much about the theoretical orientations that characterize the field. At the methodological level, this paper introduces, in the field of psychotherapy research, a quantitative method of content analysis widely used in various domains of investigation (i.e., social and behavioral sciences, technology, engineering), due to its efficiency in detecting the structure and dynamics of scientific production (Callon, Law, \& Rip, 1986; He, 1999; Nederhof \& van Wijk, 1997; Rotto \& Morgan, 1997). At the practical level, the map of the journals' thematic orientation provides an informative picture which may be of use to scholars when deciding to submit papers. 
Table 1. Journals in analysis and number of articles retrieved for each year

\begin{tabular}{|c|c|c|c|c|c|c|c|c|}
\hline Journals & Classification & 2005 & 2006 & 2007 & 2008 & 2009 & 2010 & 2011 \\
\hline Annual Review of Clinical Psychology & Transversal & 24 & 19 & 17 & 16 & 20 & 25 & 20 \\
\hline Behavior Therapy & Specialized & 41 & 39 & 36 & 38 & 38 & 52 & 67 \\
\hline Behavioral Research Therapy & Specialized & 117 & 139 & 268 & 131 & 154 & 162 & 122 \\
\hline Behavioural and Cognitive Psychotherapy & Specialized & 44 & 47 & 58 & 69 & 47 & 47 & 47 \\
\hline British Journal of Clinical Psychology & Transversal & 47 & 45 & 41 & 33 & 33 & 37 & 33 \\
\hline Clinical Psychology and Psychotherapy & Transversal & 42 & 39 & 46 & 40 & 42 & 49 & 52 \\
\hline Clinical Psychology Review & Transversal & 52 & 60 & 59 & 95 & 61 & 84 & 107 \\
\hline Clinical Psychology: Science and Practice & Transversal & 56 & 52 & 47 & 43 & 50 & 39 & 41 \\
\hline Cognitive Therapy Research & Specialized & 46 & 52 & 58 & 59 & 67 & 58 & 63 \\
\hline Family Process & Specialized & 34 & 35 & 46 & 38 & 41 & 38 & 39 \\
\hline International Journal Group Psychotherapy & Specialized & 35 & 33 & 37 & 30 & 35 & 34 & 45 \\
\hline Journal of Clinical Psychology & Transversal & 136 & 122 & 101 & 102 & 104 & 95 & 110 \\
\hline Journal of Consulting and Clinical Psychology & Transversal & 126 & 119 & 103 & 106 & 106 & 92 & 87 \\
\hline Psychoanalytic Psychology & Specialized & 43 & 56 & 63 & 47 & 30 & 31 & 31 \\
\hline $\begin{array}{l}\text { Psychology and Psychotherapy: Theory, } \\
\text { Research and Practice }\end{array}$ & Transversal & 33 & 43 & 43 & 31 & 32 & 30 & 32 \\
\hline Psychotherapy & Transversal & 49 & 50 & 52 & 44 & 48 & 57 & 52 \\
\hline Psychotherapy Research & Transversal & 45 & 58 & 68 & 68 & 68 & 65 & 56 \\
\hline
\end{tabular}

\section{Method}

\section{Sample}

The analysis adopted a cluster sampling. A sample of 17 Journals (Table 1) taken to be representative of the whole area of investigation were selected. Articles published in such Journals in the period 2005-2011 were selected. We considered this period wide enough in order to provide a reliable picture of the main topics characterizing the current state of the field. Commentaries, brief notes, and book reviewers were not included.

Journals were selected in accordance with the following procedure. First, we assumed as our universe the 104 Journals indexed in the subject category "Clinical Psychology" of the 2010 Journal of Citation Indexes. Second, the four authors of the current paper classified these Journals into three categories: transversal, specialized and not relevant. A Journal was considered transversal if: a) It publishes articles of interest for psychotherapy research; b) Such articles are framed in different theoretical orientations. A Journal was classified specialized if: a) It publishes articles of interest for psychotherapy research; b) Such articles are framed within a specific theoretical-clinical orientation/approach (e.g., cognitive therapy, psychoanalysis, group Therapy, etc.). Journals that are listed within the subject category "Clinical Psychology", yet without having psychotherapy research among their aims were considered non relevant (e.g., Neuropsychology). Third, we selected the 10 transversal Journals, with the highest 2010 Impact Factor ${ }^{1}$. Finally, we integrated this list with specialized Journals, limiting the selection to those having an Impact Factor higher than the median of the subject category. Thus, 7 Journals were selected: 4 with a cognitivebehavioural orientation, 1 focusing on family therapy, 1 with a psychoanalytic orientation and 1 focusing on group therapy ${ }^{2}$.

As a result of the procedure of sampling described above, 7,086 articles were collected. Table 1 shows the Journals under analysis and the distribution of the articles through them.

The analysis focused on the keywords indexing articles. Both keywords defined by authors and independently by SCOPUS were retrieved. A set of 5,516 keywords was composed, corresponding to 174,335 occurrences (token/type ratio: 31.6 ; about 0.77 types for article, corresponding to more than 24 keywords token for article).

\footnotetext{
${ }^{1}$ We are aware of the current political, cultural and social debate about strengths and limitations of the Impact Factor (inter alia: Hirsch, 2007; Jarvey, Usher, \& McElroy, 2012). On the other hand, so far the Impact Factor has been widely used, especially as an index for ranking Journals. Moreover, our use of the Impact Factor has been moderated by a qualitative criterion (i.e., the inclusion of specialized Journals).

${ }^{2}$ The classification was carried out according to the following procedure. First, each judge classified Journals independently. In order to attribute a Journal to a category the agreement of at least three out of four judges was required. In the (few) cases in which independent classifications presented a lower level of convergence, agreement was reached through discussion.
} 
We assumed keywords were a reliable index of the article's thematic content. Even if some authors warn about such faith, the use of keywords as a synthetic way of indexing the content of scientific production is widespread (for a discussion on the point, see Whittaker, 1989). Moreover, while it may raise some criticism when adopted for the sake of specific tasks of data retrieval (e.g., for selecting specific articles), it is worth considering less problematic when, as here, it is used to define a global picture of the whole literature. On the other hand, the choice of integrating the keywords proposed by authors with the ones provided by SCOPUS according to a systematic computational procedure, should further limit the risks of unreliability.

\section{Data Analysis}

In order to reduce the data matrix's dispersion, only the most frequent keywords were retained for the following analysis. To this end, we limit analysis to keywords corresponding to more than $50 \%$ of the whole occurrences. In so doing, we restricted the analysis to 108 keywords (corresponding to 88,801 occurrences).

The analysis was performed through a two-step procedure.

First, in order to identify patterns of co-occurring keyword, a cluster analysis (CA) was carried out. CA was applied on the data matrix having the 108 keywords as rows and the 17 Journals as columns; each $i j$ th cell reported the relative frequency of the $i$-th keyword in the $j$-th Journal. CA led to group keywords in sets of maximum inner homogeneity and maximum outer divergence. Each cluster may thus be interpreted as identifying one specific topic, as it is depicted by a particular aggregation of co-occurring keywords.

Second, clusters of keywords extrapolated by the CA were used as criterion for detecting the relations among Journals. To this end, a factorial analysis (principal components method) was performed on the matrix Journals (rows) $x$ clusters of keywords (columns), with the $i j$-th cell showing the relative frequency with which the $j$-th cluster occurred in the $i$-th Journal. Factors extracted were interpreted as semantic dimensions of similarity/dissimilarity, making the Journal's orientation among topics easier to establish.

\section{Results}

\section{Cluster Analysis}

The CA defined 8 clusters as optimal partition of the set of keywords. Yet, as an effect of the highly dispersive distribution of data (i.e., most of the cells reported 0 or very low score) this partition proved to have little significance: 6 clusters have less than $3 \%$ of keywords (cluster 1, 2, 3, 4, 5, 7), one cluster (cluster 6) about $11 \%$, and one cluster (cluster 8)

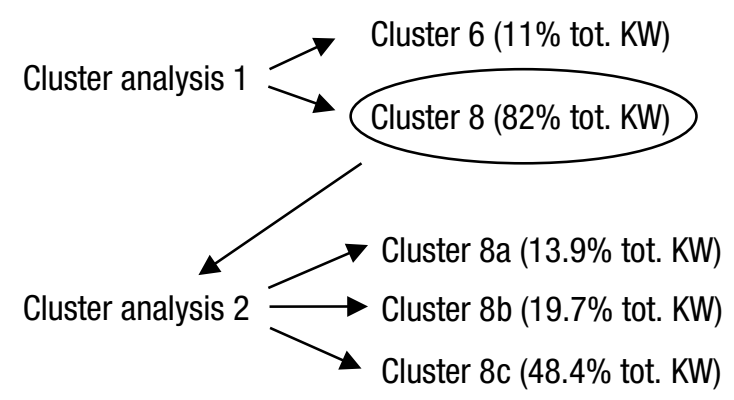

Figure 1. The most representative clusters identified by the two cluster analysis. KW=Keywords.

about $82 \%$. Therefore, we performed a second CA on the keywords grouped in cluster 8 . The second CA broke cluster 8 down into 3 sub-clusters (Figure 1). Thus, as result of the combination of the two CAs, we considered a partition of 4 clusters of keywords, classifying about $93 \%$ of the original set of keywords: cluster 6 , cluster $8 \mathrm{a}, 8 \mathrm{~b}$, and $8 \mathrm{c}$ (Table 2). Each cluster was interpreted as a topic and labelled according to the meaning of the most representative keywords composing it.

Topic A. Cognitive and behavior treatments. This topic corresponds to cluster 6 of the first CA ( $11 \%$ of the 108 keywords). It aggregates keywords referring to cognitive and behavior therapy (Cognitive and Behavioral therapy). Other keywords mark targets of the treatment (Depression, Anxiety Disorder, Adolescent) and the model of research adopted (Major clinical study, Controlled study).

Topic B. The study of mental disease. This corresponds to cluster 1 of the second CA (13.9\%). The most frequent subgroup of keywords of this cluster concerns mental disease (Mental Disease, Comorbidity, Obsessive Compulsive Disorder, Post Traumatic Stress Disorder). Other keywords refer to the type of research and its instruments (Clinical trial, Clinical article, Self-report). Thus, we are led to interpret the cluster as indicative of the focus on the (clinical and/or experimental) investigation of psychopathology.

Topic c. Intervention on severe mental disorders. This corresponds to cluster 2 of the second CA (19.7\%). The most frequent subgroup of keywords concerns field conditions and contextual aspects of interventions (Social support, Risk factor, Family, Prevalence, Doctor patient relation). Other keywords define the psychopathological area of interest, in particular defined by psychotic disorders (DSM, Mental Disorders, Schizophrenia, Bipolar Disorders). Accordingly, we are led to interpret the cluster as indicative of the focus on interventions on severe disorders (psychosis, personality disorders). This kind of intervention involves contextual dimensions and encompasses several levels of analysis/stand- 
4 The topics of psychotherapy research

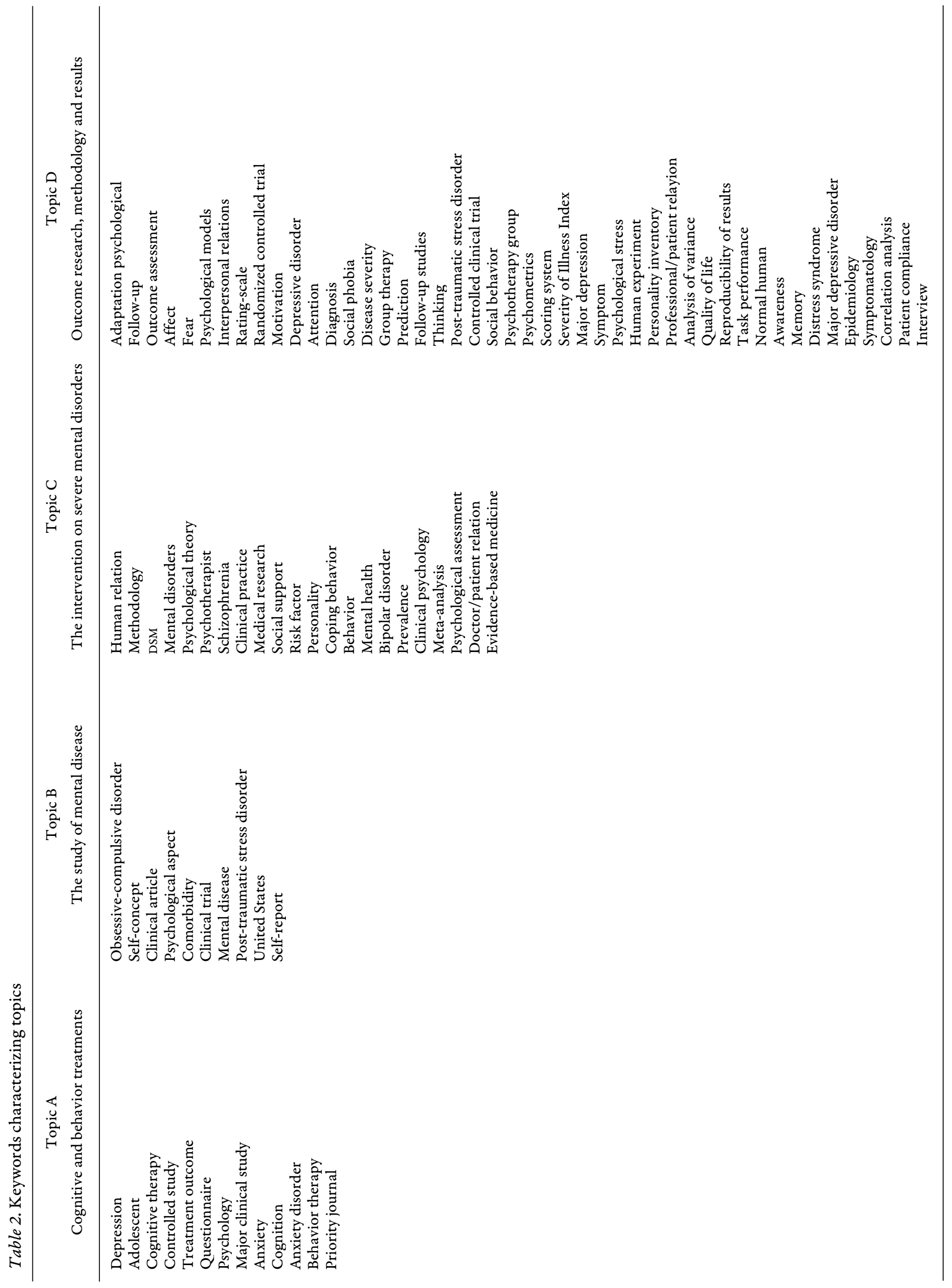


Table 3. Relative frequency of the occurrences of each topic for each journal

\begin{tabular}{lcccc}
\hline \multicolumn{1}{c}{ Journals } & Topic A & Topic B & Topic C & Topic D \\
\hline Annual Review of Clinical Psychology & .231 & .138 & .332 & .220 \\
Behavior Therapy & .324 & .096 & .046 & .262 \\
Behavioral Research Therapy & .332 & .086 & .057 & .262 \\
Behavioural and Cognitive Psychotherapy & .395 & .121 & .080 & .218 \\
British Journal of Clinical Psychology & .297 & .085 & .082 & .283 \\
Clinical Psychology and Psychotherapy & .315 & .121 & .097 & .239 \\
Clinical Psychology Review & .287 & .134 & .231 & .253 \\
Clinical Psychology: Science and Practice & .261 & .171 & .334 & .184 \\
Cognitive Therapy Research & .386 & .084 & .058 & .245 \\
Family Process & .140 & .181 & .173 & .158 \\
International Journal Group Psychotherapy & .177 & .128 & .166 & .370 \\
Journal of Clinical Psychology & .274 & .131 & .154 & .193 \\
Journal of Consulting and Clinical Psychology & .343 & .052 & .072 & .267 \\
Psychoanalytic Psychology & .185 & .164 & .319 & .215 \\
Psychology and Psychotherapy: Theory, & .282 & .102 & .120 & .235 \\
Research and Practice & .245 & .109 & .278 & .191 \\
Psychotherapy & .321 & .096 & .134 & .231 \\
Psychotherapy Research & & &
\end{tabular}

Note. Topic A = Cognitive and behavior treatments; Topic B = The study of mental disease; Topic C = The intervention on severe mental disorders; Topic $\mathrm{D}=$ Outcome research, methodology and results.

points (Psychological theory, Clinical psychology, Evidence based medicine) as well as forms/logics of action (Clinical psychology, Evidence based medicine) as well as forms/logics of action (Clinical practice; Medical research; Psychological assessment) thus giving the topic the appearance of a boundary theme between psychotherapy and the broader domain of clinical psychology.

Topic D. Outcome research, methodology and results. This corresponds to cluster 3 of the second CA (48.4\%). Several keywords mark articles devoted to the experimental ( $R C T$, Controlled clinical trial) evaluation of the outcome (Follow-up, Followup studies; Outcome assessment, Prediction). Other keywords seem to be markers of studies with a methodological focus (e.g., Rating scale, Psychometrics, Severity of Illness Index, Scoring-system, Personality inventory, ANOVA, Correlation analysis). Most of the other keywords specify goal and content of the studies, in terms of clinically relevant crite$\mathrm{ria} /$ targets of evaluation as well as factors involved (e.g., Psychological adaption; Affect, Fear, Thinking, Motivation, Attention, Social behavior, Interpersonal relation, Quality of Life). No reference is made to specific models of psychotherapy.

\section{Factorial Analysis}

Table 3 shows the distribution of the 4 topics across the 17 Journals. As we said, this matrix was the one subjected to factorial analysis (FA). FA led to the extraction of two main components, accounting for $84.6 \%$ of the total variance.

Table 4 shows the topics' coordinates on factor dimensions (the higher the coordinate, the stronger the correlation between the topic and the factorial dimension). A right polarity of the first factor is associated with topic A (Cognitive and behavior treatments) and, to a lesser degree, topic $\mathrm{D}$ (Outcome research, methodology and results); the opposed polarity is associated with topic B (The study of mental disease) and topic $\mathrm{C}$ (The intervention on severe mental disorders).

Accordingly, we interpret the first factor as a marker of a semantic dimension concerning the aims of scientific works published by the Journals. This dimension is depicted by the opposition between a focus on treatments and their efficacy versus a focus on the study of psychopathology. Needless to say the two foci are not conceptually alternative. Nevertheless, they appear to work as an oppositional relationship defining a kind of figure/background shift. On the one hand the empirical validation of the psychotherapy seems to be the central interest (and with it the associated methodological issues too); on the other hand, psychotherapy-and more in general the clinical intervention where psychotherapy and contextual dynamics are intertwined-as a process aimed at dealing with psychopathology and promoting mental health. We 
Table 4. Topics' coordinates for each factor

\begin{tabular}{lcc}
\hline & Factor 1 & Factor 2 \\
\hline Topic A & .81 & -.48 \\
Topic B & -.92 & .00 \\
Topic C & -.88 & -.07 \\
Topic D & .56 & -.81 \\
\hline
\end{tabular}

Note. Topic A = Cognitive and behavior treatments; Topic $\mathrm{B}=$ The study of mental disease; Topic $\mathrm{C}=$ The intervention on severe mental disorders; Topic $\mathrm{D}=$ Outcome research, methodology and results.

summarize this semantic opposition in the labels of the polarities: Validation of models of treatment versus Management of intervention on disease. As one can see, this distinction somehow recalls the classical product/process division. Nevertheless, we prefer not to use the latter in order to avoid confusion between this well-established representation of the literature and the results of our analysis.

As concerns the second factor dimensions, one polarity (up) is associated with topics A (Cognitive and behavior treatments), while the other (bottom) is associated with topic $\mathrm{D}$ (Outcome research, methodology and results). Accordingly, we interpret it as a dimension concerning the extension of the Journals' target of study. This dimension is characterized by the contrast between two approaches: an approach targeted on a specific clinical orientation versus an approach having a more general interest. Thus, we label the up polarity Restricted target and the bottom polarity Generalized target.

Factorial analysis results allow us to detect the association between factors and also Journals. We represent such relationship in geometrical terms. To this end we refer to the transformation of the measures of association in coordinates on the bidimensional space defined by the two factorial dimensions. Figure 2 depicts the semantic frame thus defined and the position of Journals within it. In the final analysis, this semantic frame can be seen as the spatial representation of the similarity/dissimilarity among Journals, as to the topics characterizing them (the closer two Journals on the bi-dimensional space, the more similar they are, as to the topics of articles they publish).

As one can see, most Journals (the only exceptions are International Journal of Group Psychotherapy and Behavioural and Cognitive Psychotherapy) lie along the first dimension where they can be grouped in three broad classes. A group of 5 Journals (Journal of Consulting and Clinical Psychology, Behavior Research and Therapy; Behavior Therapy; Cognitive Therapy Research; British Journal of Clinical Psychology) is placed close the Validation of models of treatment polarity; it contrasts with a group of 4 Journals (Family process; Psychoanalytic psycholo-

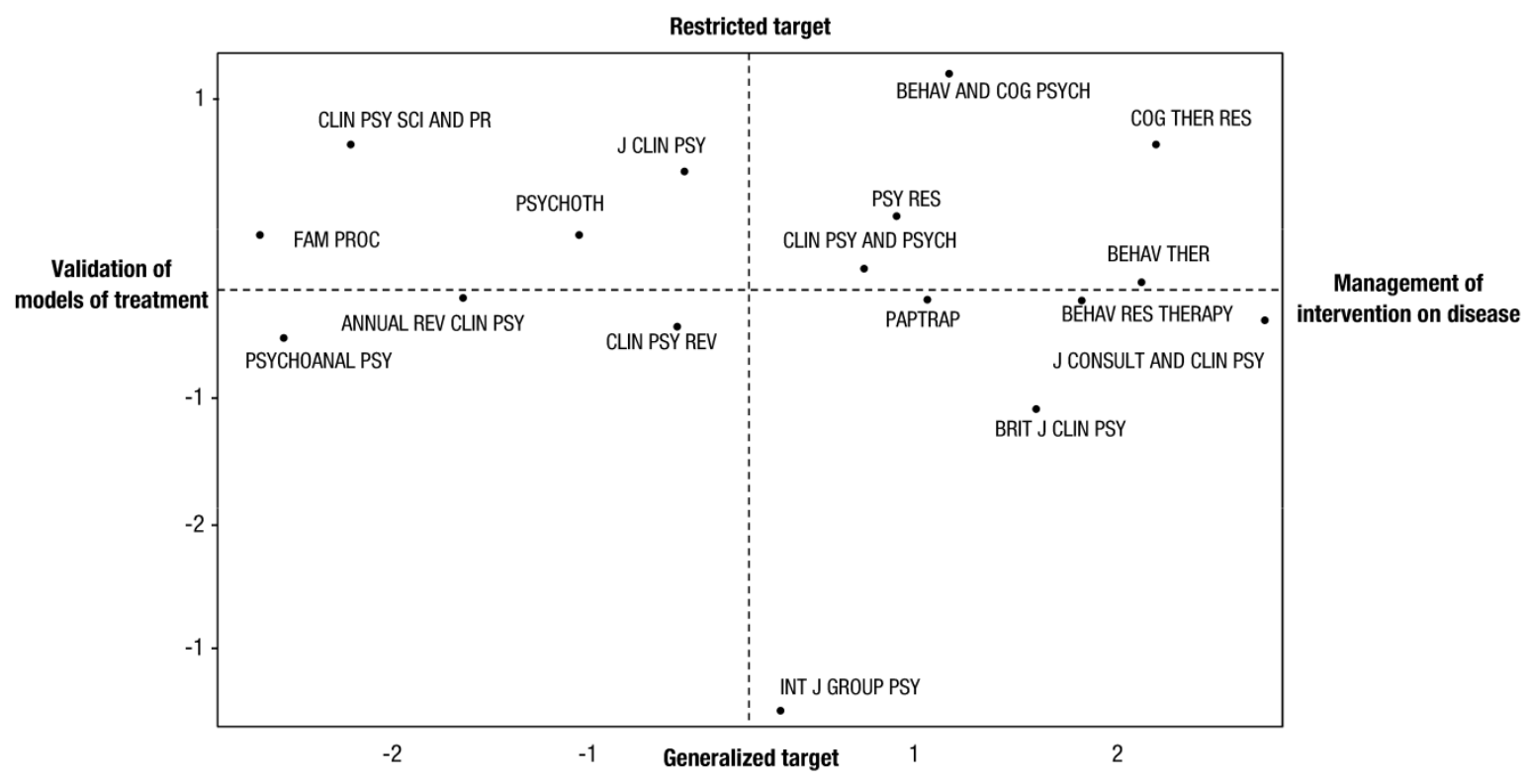

Figure 2. Factorial space and journals' positioning. ANNUAL REV CLIN PSY = Annual Review of Clinical Psychology; BEHAV THER = Behavior Therapy; BEHAV RES THERAPY = Behavioral Research Therapy; BEHAV AND COG PSYCH $=$ Behavioural and Cognitive Psychotherapy; BRIT J CLIN PSY = British Journal of Clinical Psychology; CLIN PSY AND PSYCH = Clinical Psychology and Psychotherapy; CLIN PSY REV = Clinical Psychology Review; CLIN PSY SCI AND PR = Clinical Psychology: Science and Practice; COG THER RES = Cognitive Therapy Research; FAM PROC = Family Process; INT J GROUP PSY = International Journal of Group Psychotherapy; J CLIN PSY = Journal of Clinical Psychology; J CONSULT AND CLIN PSY = Journal of Consulting and Clinical Psychology; PSYCHOANAL PSY = Psychoanalytic Psychology; PAPTRAP = Psychology and Psychotherapy: Theory, Research and Practice; PSYCHOTH = Psychotherapy; PSY RES = Psychotherapy Research. 
gy; Annual Review of Clinical Psychology, Clinical Psychology: Science and Practice) associated with the Management of intervention on disease polarity. In the middle, the other 7 Journals (Psychology and Psychotherapy: Theory, Research and Practice; Clinical Psychology and Psychotherapy; Psychotherapy Research; Clinical Psychology Review; Journal of Clinical Psychology; Psychotherapy) positioned around the origin of the axis, therefore to be interpreted as less characterized by the polarities, namely more pluralistic as to topics of interest.

\section{Discussion}

The results presented deserve some comments. Firstly, it is worth noting the way keywords are distributed over articles. The number of types $(5,516)$ is rather high once compared with the number of articles-on average, any type of keyword has the probability of occurring not far from 1 (every 0.77 articles). On the other hand, this datum is the effect of a very asymmetrical distribution: 108 out of 5,516 types (2\%) correspond to half of the total occurrences. Thus, according to the picture drawn from the keywords used by authors, psychotherapy research proves to be a quite heterogeneous field, characterized by a very restricted semantic core-the one detected by the most frequent keywords-and, on the other hand, by a very broad collection of specific, quantitatively marginal contents. Two interpretations of this structure are possible. (a) It might reflect the fragmentation of the research interests across the field. According to this hypothesis, the dispersion of the keywords' distribution might be the result of the high heterogeneity of objects and aims informing the scientific practices in the field. (b) The dispersive distribution might be the effect of a linguistic idiosyncrasy in choosing keywords, namely the fact that different authors use different keywords to refer to the same contents. Needless to say, the two interpretations are not alternative; rather, they could work complementarily. Only further analyses will enable us to understand their importance better. Nevertheless, in any case the distribution of keywords provides a clue that leads to see psychotherapy research as a scientific field that is still far from the homogeneity and unitedness (in terms of aims, objects, shared linguistic codes) characterizing the paradigmatic context of scientific enterprises (Kuhn, 1962).

Second, topics extrapolated by the CA provide a picture of the field which is consistent with the common ground representation. Topics seem to reflect the anchoring to three basic semantic references that any clinical researchers would recognize as salient conceptual and pragmatic organizers of the field. As reflected in Topic $\mathrm{D}$, one basic anchoring point is provided by the theme of the evaluation of treatments' effects. We need not spend time pointing out that this theme is at the foundation of psychotherapy research-any history of such a field makes it begin with pioneering studies on the effect of psychotherapy. As topic A (Cognitive and behavior treatments) shows, a second anchoring point is provided by the commitment to cognitive-behavioural therapy. Though the extrapolation of topics is not necessarily a marker of quantitative prevalence, it is worth noting the fact that this is the only clinical orientation that is able to "coagulate" a specific topic. We are led to interpret this datum as an indication of the relevance that scientific production on cognitive-behavioural therapy has acquired within the field. Needless to say, the fact that cognitive-behavioural therapy has proved to be able to coagulate a specific topic is, at least partially, the effect of the composition of the sample adopted ( 4 out 17 Journals were specialized Journals focused on the area of cognitive-behavioural therapy). Yet, the latter observation does not reduce the value of the result at stake. Rather, it adds a further significance to it, namely it highlights how the importance achieved by the cognitive-behavioural approach reflects its capacity to interpret-and therefore be prized by-the current scientific standards of clinical research, the ones reflected in the bibliometric criterion of sampling adopted. Psychopathology represents the third semantic organizer, grounding topics B (The study of mental disease) and $\mathrm{C}$ (The intervention on severe mental disorders). Here it is also interesting to observe the differentiation between disease and disorders, which marks the linguistic boundary between topic B and topic C. In the context defined by the co-occurring keywords sustaining the topics, this boundary seems to be something more than a mere linguistic variation. Rather, it seems to be grounded on a semantic context: According to our interpretation, topic B (The study of mental disease) seems to be focused on less severe forms of psychopathology and their treatment, while topic C (The intervention on severe mental disorders) seems to be focused on a broader view of the intervention, encompassing contextual issues as well as addressing more severe psychopathological conditions. Incidentally, this can be interpreted as a sign of a weaker linguistic interpretation of the dispersive distribution of keywords (see above): Insofar as the disease-disorders variation seems to be rooted in a semantic context, it could be plausible that the same happens in the cases of other labels and their variation. Finally, it must be mentioned that this third semantic organizer-psychopathology - may have emerged as a result of a sample bias. As a matter of fact, the procedure of sampling we adopted has filtered Journals, rather than articles in terms of relevance to the psychotherapy research field. Therefore, the set of articles analyzed encompassed a proportion of articles not concerned specifically with psychotherapy research, even though published in Journals ( 9 out of 17) having psychotherapy research among their aims. Only fur- 
ther analyses will allow to check to what extent the presence of this kind of articles has contributed to making topics $\mathrm{B}$ and $\mathrm{C}$ emerge.

Third, the semantic space modeled by the twostep multidimensional analysis proves to be grounded basically on the opposition between interest in the Validation of models of treatment and interest in the Management of intervention. As a matter of fact, it is along this dimension that Journals define their reciprocal relationships. Our thesis, which will have to be tested in further analysis, is that such a semantic opposition goes beyond the process/outcome distinction, and more generally concerns the dialectics between an interest focused on the scientific legitimation of the psychotherapy and an interest focused on the understanding/empowering of psychotherapy as a device for addressing clinical issues. These two foci are historically found throughout the scientific cultures and practices within the psychotherapy field; they are not conceptually alternative-yet it is hard to act as if they were immediately complementary (Salvatore et al., 2010).

Fourth, the relationship between topics and Journals lends itself to being interpreted as supporting the distinction we adopted between specialized and transversal Journals. Specialized Journals have the highest level of association with the factorial dimension-namely they tend to be more specific as to topics of interest. On the other hand, they do not appear to work as a separate subset-rather, even if 4 Journals committed to cognitive-behavioral therapy lie close to each other, all but one of the specialized Journals (International Journal of Group Psychotherapy) have relationships of similarity with transversal Journals too. Thus, one can conclude that our classification of Journals as specialized is consistent with their semantic content and yet that their inclusion in the sample did not distort the sample; rather it allowed us to encompass a semantic area which otherwise would have been marginalized.

Finally, if one wants to draw a synthetic picture of the Journals' thematic orientations, one can conclude that in the final analysis they can be grouped in three general classes: A class of Journals that prefer to host outcome research, aimed at the validation of models of treatments; a class of Journals with a more general orientation, which means aspects concerning psychotherapy are integrated and projected on a broader domain of clinical-psychological interest-a domain where the focus moves (or is extended) to the management of interventions, as depending on contextual and processual factors. In the middle, a class of generalist Journals, namely Journals that are sensitive to both the above orientations, and so are not characterized by either of them.

Some specific limits of the current study must be mentioned, because they limit the conceptual breadth of the findings. First, as has already been said, the criterion of definition of the universe of analysis adopted is the fact of being published in a
Journal aimed also at psychotherapy research. Consequently, it was possible to discriminate articles not specifically concerned with psychotherapy research, yet published in Journals committed, inter alia, to this area. Second, keywords are a significant clue of articles' content. Yet they provide a rather poor, generic representation of it. As highlighted, this limit has prevented us from reaching a reliable interpretation of the semantic structure underpinning the way keywords are distributed among articles. Third, associated with the previous point, it has to be recognized that the current study suffers from the absence of a distinction between keywords defined by authors and defined by independent judges (i.e., provided by SCOPUS). While the use of both is a way of empowering the reliability of this index, the absence of distinction between them removes a major source of information. Anyway, the greatest limit to the current study lies in the structure of data it is based on. The two-step multidimensional analysis was performed on two data matrixes: a data matrix defined by keywords (rows) and Journals (columns) and a data matrix defined by Journals (rows) and clusters (columns). This structure of data defines the meaning of findings. In particular, findings concern the co-occurrence of keywords in the context of Journals (first step) and topics (second step). This means that the topics extrapolated concern semantic nuclei that take shape at the level of the grouping of Journals, rather than articles. And the same can be said for the second step: the semantic dimensions identified model the relationship among Journals compared to topics, and therefore they cannot be considered a snapshot of the semantic structure of articles.

Despite the limits and the questions raised by the study, it seems to us that the picture of the psychotherapy research field it provides is worthwhile. As the discussion of the findings has showed, the analysis of the semantic structure of the psychotherapy research provides food for thought, highlighting relevant epistemological and theoretical issues (e.g., the unitary nature of the area, the relationship between models of publication and topic's centrality) and at the same time contributing to address them. Moreover, this study has showed that a qualiquantitative method of content analysis, where multidimensional techniques of data analysis ground and support the researcher's interpretative job, rather than substitute it, can provide a meaningful picture even of a quite complex scenario like psychotherapy research. A picture, moreover, that already at the current level of definition may provide hints about the Journals' scientific-cultural politics-a rather important issue with pragmatic implications at the institutional and individual level.

Further studies will try to make progress in the direction of investigation opened by the current work. In particular, we see several objectives that need to be pursued: 
a) The move to a more detailed level of analysis, centred on articles, thus identifying patterns of keywords as co-occurring within the same article.

b) The introduction of a longitudinal standpoint, thus analysing the evolution over time of topics and semantic structure of the field.

c) The introduction of the abstract as a further source of information-thus increasing the "resolution" of the analysis as well as to test the reliability of keywords.

d) The enlargement and specification of the universe of analysis, thus increasing its consistency with what the scientific community considers to be psychotherapy research.

\section{Conclusion}

This study reports the findings of an analysis of keywords indexing the content of articles published in Journals operating in the psychotherapy research field. The analysis, based on a two-step multidimensional procedure, provided a map of the contents characterizing such a field as well as of how Journals orient their interests towards them. Four main topics were extrapolated, interpreted as the expression of three basic semantic organizers: cognitive-behavioural therapy, outcome evaluation and psychopathology. Topics and their semantic organizers give shape to a semantic space modelled in terms of two semantic dimensions: one concerning the articles' subject and the other concerning with the extension of the target. The former is structured in terms of the opposition between two general aims: the management of the intervention versus the validation of models of treatments; the latter is structured in terms of the opposition between a restricted and a generalized target of research. However, the Journals analyzed proved to differ mainly in terms of the first semantic dimension detected. According to it, Journals can be grouped in three large classes: a group of Journals pursuing the validation of models of treatment as main scientific-cultural interest; a group more interested of the themes concerned with the development of interventions and their management; a middle group composed of Journals whose commitment integrates both interests.

Before concluding, it has to be said that the map provided by the study must not be intended as an objective, detailed representation of the ever-changing scenario of psychotherapy research. Rather, it has to be seen as an interpretative device useful for deepening the understanding of the semantic organization underpinning the current status of research in the field. This is so because content analysis, like any kind of analysis concerning meanings, is inherently abductive and interpretative, also when, as in the case of the current study, it adopts a quantitative method (Salvatore, Gennaro, Auletta, Tonti, \& Nitti, 2012).

\section{References}

Callon, M., Law, J., \& Rip, A. (Eds). (1986). Mapping the dynamics of science and technology: Sociology of science in the real world. London: The Macmillan Press Ltd.

$\mathrm{He}$, Q. (1999). Knowledge discovery through co-word analysis. Library Trends, 48(1), 133-159.

Hirsch, J.E. (2007). Does the h-index have predictive power? Proceedings of the National Academy of Sciences, 104(49), 19193-19198.

Jarvey, P., Usher, A., \& McElroy, L. (2012). Making research count: Analyzing Canadian academic publishing cultures. Toronto: Higher Education Strategy Associates.

Kuhn, T. S. (1962). The structure of scientific revolutions. Chicago: University of Chicago Press.

Manzo, S. (2010). La ricerca di processo nel decennio 19982007: Contributo a una review critica [Process research in the 1998-2007 decade: A contribute for a critical review]. Ricerca in Psicoterapia/Research in Psychotherapy, 1(13), 92-119. Retrieved from http://www.researchinpsychotherapy.net

Nederhof, A. J., \& van Wijk, E. (1997). Mapping the social and behavioural science world-wide: Use of map in portfolio analysis of national research efforts. Scientometrics, 40(2), 237-276.

Rotto, E., \& Morgan, R. P. (1997). An exploration of expertbased text analysis techniques for assessing industrial relevance in U.S. engineering dissertation abstracts. Scientometrics, 40(1), 83-102.

Salvatore, S. (2011). Psychotherapy research needs theory. Outline for an epistemology of the clinical exchange. Integrative Psychological and Behavioral Science, 45(3), 366-388.

Salvatore, S., Gennaro, A., Auletta, A., Grassi, R., Nitti, M., Manzo, S., ... Gelo, O. (2010). La psicoterapia come scambio comunicativo. Prospettive di ricerca sul processo clinico. [Psychotherapy research as communicative exchange. Research perspectives in clinical process] Ricerche in Psicoterapia/Research in Psychotherapy, 13(2), 241-286. Retrieved from http://www.researchinpsychotherapy.net

Salvatore, S., Gennaro, A., Auletta, A., Tonti, M., \& Nitti, M. (2012). Automated method of content analysis. A device for psychotherapy process research. Psychotherapy Research, 22(3), 256-273.

Whittaker, J. (1989). Creativity and conformity in science: titles, keywords and co-word analysis. Social Studies of Science, 19, 473-496.

Received August 6, 2012 Revision received September 20, 2012 Accepted September 25, 2012 